\title{
Photometric Studies of Bright Southern Binary Systems: $\epsilon$ Cra and $\psi$ Ori
}

\author{
R. R. Shobbrook $\cdot$ S. Zola
}

Received: 1 November 2005 / Accepted: 1 February 2006

(C) Springer Science + Business Media B.V. 2006

\begin{abstract}
We present results from detailed analyses of new data combined with previously published light curves of $\epsilon$ Cra and $\psi$ Ori. Based on the shape of the secondary minimum of $\epsilon \mathrm{CrA}$ we found that the discrepancy between the photometric and spectroscopic mass ratio, although marginal, is statistically significant. We propose a third light as a possible solution and derive the absolute parameters of components. The physical parameters of components of the early-type binary system $\psi$ Ori were also obtained from the light curve modelling. Our solution indicate that $\psi$ Ori is a detached, grazing-eclipse system.
\end{abstract}

Keywords Binaries: eclipsing, Binaries: close, Binaries: contact, Stars: Fundamental parameters

\section{Introduction}

Between 1991 and 2001 many observations were obtained of bright eclipsing or ellipsoidal binary stars which may also be observed with the Sydney University Stellar Interferometer, SUSI. The purpose of this program was to obtain photometry to enable some parameters to be fixed during the complex analysis of the SUSI data. The observations were taken using the 24-inch telescope of the Australian National University at Siding Spring Observatory (hereafter referred to as SSO).

\section{R. R. Shobbrook}

Research School of Astronomy and Astrophysics, Australian

National University, Weston Creek P.O., ACT 2611, Australia

\section{S. Zola $(\bowtie)$}

Astronomical Observatory of the Jagiellonian University, ul. Orla 171, 30-244 Cracow, Poland; Mt. Suhora Observatory, Pedagogical University, ul. Podchorazych 2, 30-084 Cracow, Poland
The new data will be referred to as $S S O V$ magnitudes. The details of the observing programme and the equipment is given by Shobbrook (2004).

$\epsilon \mathrm{CrA}$ (HD155813) is the brightest $\left(4.73^{\mathrm{m}}\right) \mathrm{W}$ UMatype system, with period $P=0.59^{\mathrm{d}}$ discovered by Cousins and Cox (1950). UBV observations were reported by Tapia (1969) and those in RI passbands by Hernandez (1972). The UBVRI set was analyzed with the Wilson-Devinney (W-D) code by Twigg (1979) who photometrically determined the mass ratio to be very small: $q=0.113$. The spectroscopic orbit of $\epsilon$ Cra was published by Goecking and Duerbeck (1993). The following orbital elements were obtained: $K_{1}=$ $34.5 \mathrm{~km} / \mathrm{s}, K_{2}=266.9 \mathrm{~km} / \mathrm{s}$ and $\gamma=57.9 \mathrm{~km} / \mathrm{s}$.

$\psi$ Ori (HD 35715, $V=4.6^{\mathrm{m}}$ ) was found to be a spectroscopic binary with period of 2.5 days by Frost and Adams (1903). The most recent orbital solution made by Telting et al. (2001) resulted in the following orbital elements: $K_{1}=$ $144.6 \pm 0.5 \mathrm{~km} / \mathrm{s}, K_{2}=237 \pm 4 \mathrm{~km} / \mathrm{s}, \gamma=+19 \pm 5 \mathrm{~km} / \mathrm{s}$, $e=0.053 \pm 0.001$, and $\omega=172 \pm 5^{\circ}$. The light curve of $\psi$ Ori, as shown by Percy (1969), is typical of an ellipsoidal variable. The depths of the minima in the SSO data are approximately $0.035^{\mathrm{m}}$ and $0.020^{\mathrm{m}}$. The $\mathrm{U}$ and B light curves have been analyzed by Hutchings and Hill (1971). The authors found an orbital inclination of $58 \pm 8^{\circ}$ and derived the absolute parameters: $M_{1}=14.2 M_{\odot}, M_{2}=8.6 M_{\odot}, R_{1}=$ $6.1 \mathrm{R}_{\odot}, R_{2}=4.7 R_{\odot}$.

\section{Results from the light curve modelling}

In order to obtain the physical parameters of each system we used the W-D code (Wilson, 1979) supplemented with the Monte Carlo search procedure. The theoretical values of both albedo and gravity darkening coefficients were used. 
Fig. 1 Comparison between theoretical and observed light curves of $\epsilon \mathrm{Cra}$. Observations are shown by squares and theoretical curves by lines
Fig. 2 Comparison between theoretical and observed light curves of $\psi$ Ori
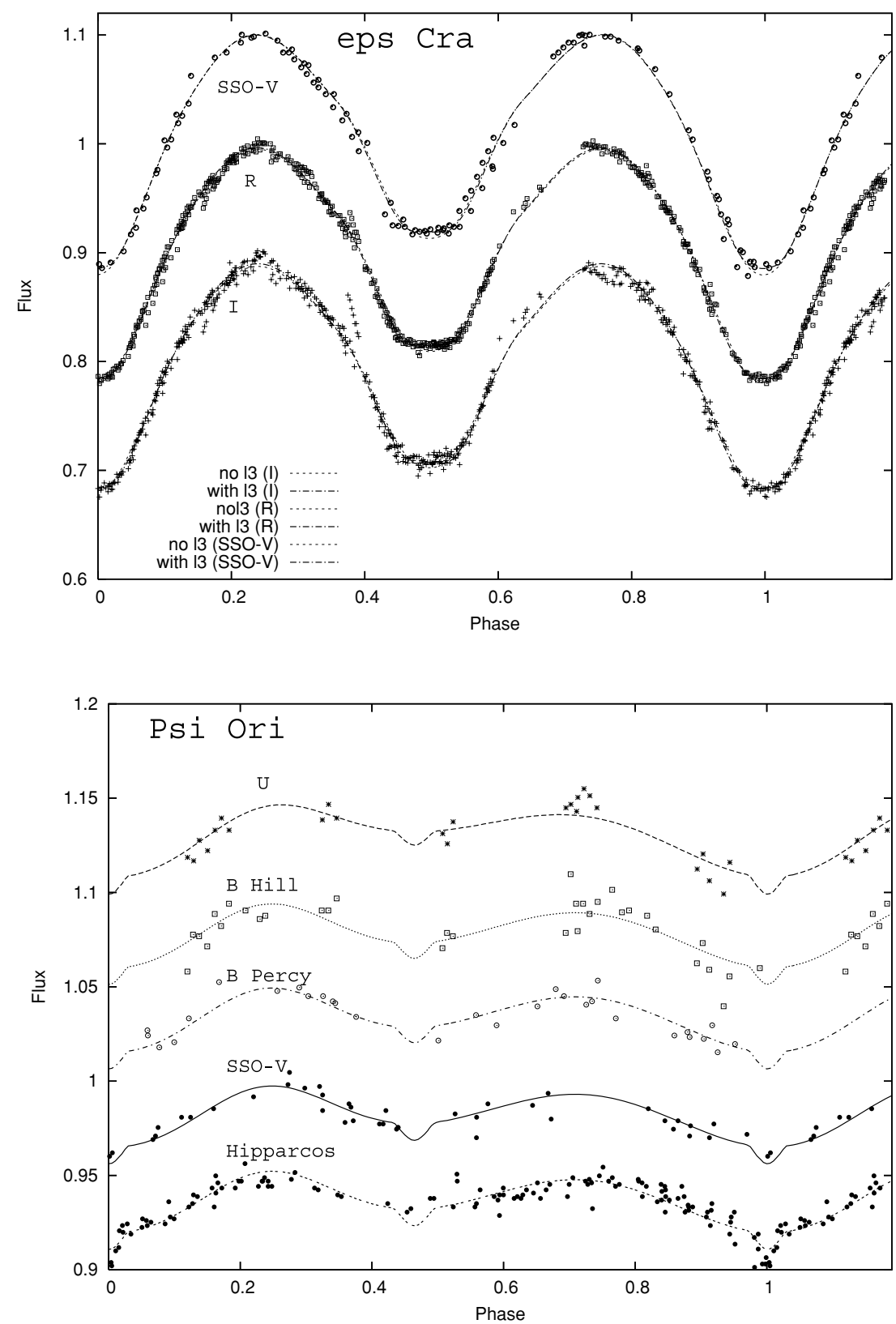

The limb darkening coefficients were adopted as functions of the temperature and wavelength from the Díaz-Cordovés et al. (1995) and Claret et al. (1995) tables. For both systems we fix two crucial parameters: the temperature appropriate for the spectral type and the mass ratio, as determined from the radial velocity curves.

The solution for $\epsilon$ CrA was made using the Hernandez (1972) RI light curves and the new SSO-V filter observations. We are obliged to point out that there appears to be a significant number of typing errors in the groups of Julian Dates of the Hernandez's data table. Most errors were able to be corrected by comparison with the Julian Dates in the Tapia (1969) UBV data tables, since their data were obtained on the same nights at the same observatory. However, there are some observations, which appear at phase close to 0.38 in Fig. 1 in the R and I curves, which are clearly in error but could not be corrected; these have not been included in the analysis discussed below.

For $\epsilon \mathrm{CrA}$, at first we fixed the mass ratio at $q=0.129$, obtained by Goecking and Duerbeck (1993) and the temperature of the primary star at 6700 . In our light curve modelling of $\psi$ Ori we used the Toronto data (U and B filters), the Kitt Peak B data, the data collected by the Hipparcos mission and the new SSO V observations. We fixed the temperature of the primary component at $26000 \mathrm{~K}$ (B1 spectral type), the mass ratio value at $q=0.61$ and the eccentricity $e=0.053$, as obtained by Telting et al. (2001). We assumed that there is no third light in this system. 
The theoretical light curve along with observations are shown in Figs. 1 and 2 for $\epsilon$ Cra and $\psi$ Ori, respectively. The final physical parameters for the components are: $M_{1}=$ $1.74 \pm 0.06 M_{\odot}, M_{2}=0.21 \pm 0.02 M_{\odot}, R_{1}=2.14 \pm 0.03$ $R_{\odot}, R_{2}=0.86 \pm 0.03 R_{\odot}$ for $\epsilon$ Cra and $M_{1}=12.09 \pm 0.35$ $M_{\odot}, M_{2}=7.37 \pm 0.18 M_{\odot}, R_{1}=4.09 \pm 0.08 R_{\odot}, R_{2}=$ $2.59 \pm 0.26 R_{\odot}$ for $\psi$ Ori.

For $\epsilon$ Cra we found the contact configuration with an fillout factor of $63 \%$. To obtain a good fit, we found it necessary either to reduce the mass ratio by 0.02 , or to postulate the existence of a third light in the system. We conclude that there is still a small discrepancy between the spectroscopic and photometric mass ratio for $\epsilon \mathrm{Cra}$ and, if there are no systematic errors in the determination of the radial velocities (especially those of the fainter componennt possibly due to line blending), it can be removed only by adding a third light.

$\psi$ Ori has a detached configuration with the primary, more massive, component being closer to its Roche lobe. Our model suggests that the light curve of $\psi$ Ori has a very shallow eclipse also observed by Waelkens and Rufener (1983). Unfortunately, the phases around the minima have not been covered by earlier $\mathrm{U}$ and $\mathrm{B}$ observations. These phases definitely deserve more observers' attention to improve the solution for this early type binary.

Acknowledgements SZ acknowledges support by the KBN grant No. 2 P03D 006 22. RRS is grateful to the Director and TAC of the Mount Stromlo and Siding Spring Observatories for the use of their facilities.

\section{References}

Claret, A., Díaz-Cordovés, J., Giménez, A.: A\&AS 114, 247 (1995)

Cousins, A.W.J., Cox, A.N.: MNASSA 9, 90 (1950)

Díaz-Cordovés, J., Claret, A., Giménez, A.: A\&AS 110, 329 (1995)

Frost, E.B., Adams, W.S.: ApJ 17, 246 (1903)

Goecking, K.-D., Duerbeck, H.W.: A\&A 278, 463 (1993)

Hernandez, C.A.: AJ 77, 152 (1972)

Hutchings, J.B., Hill, G.: ApJ 167, 137 (1971)

Lu, W.: PASP 97, 428 (1985)

Percy, J.R.: JRASC 63, 233 (1969)

Pickering, E.C.: Fifty-fourth annual report of the director of the astronomical observatory of Harvard College for 1899, p. 7 (1899)

Shobbrook, R.R.: JAD 10, 1 (2004)

Tapia, S.: AJ 74, 533 (1969)

Telting, J.H., Abbott, J.B., Schrijvers C.: A\&A 377, 104 (2001)

Twigg, L.W.: Month. Not. RAS. 189, 907 (1979)

Waelkens, C., Rufener, F.: A\&A 121, 45 (1983)

Wilson, R.E.: ApJ 234, 1054 (1979) 